\title{
Nucleotide Substitutions of Hepatitis E Virus Genomes Associated with Fulminant Hepatitis and Disease Severity
}

\author{
Jun Inoue, ${ }^{1}$ Masaharu Takahashi, ${ }^{2}$ Hitoshi Mizuo, ${ }^{3}$ Kazuyuki Suzuki, ${ }^{4}$ \\ Tatsuya Aikawa, ${ }^{5}$ Tooru Shimosegawa ${ }^{1}$ and Hiroaki Okamoto ${ }^{2}$

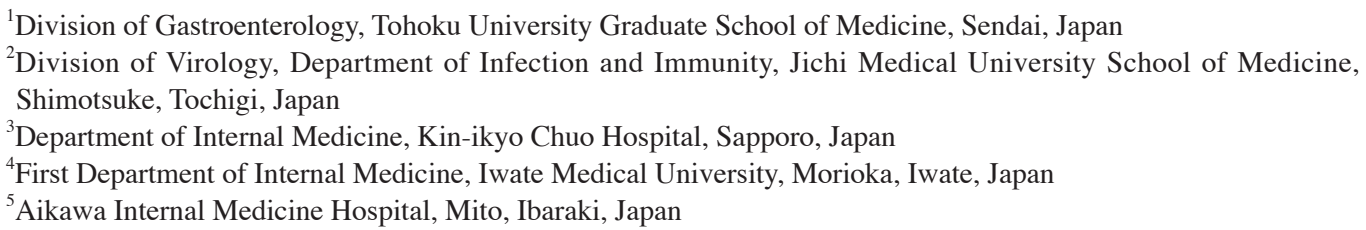

Hepatitis $E$ virus (HEV) is one of the causative agents of acute or fulminant hepatitis. Viral factors may play a role in the pathogenesis of fulminant hepatitis $\mathrm{E}$. We aimed to investigate the nucleotide substitutions of the HEV genome affecting the severity of hepatitis E. The comparison of 28 reported full-length nucleotide sequences of genotype $4 \mathrm{HEV}$ showed that the substitution of $\mathrm{C}$ at nucleotide 5907 (C5907) was most closely associated with fulminant hepatitis (fulminant hepatitis, $100 \%$; acute hepatitis, $39.1 \% ; p=0.0204$ ). Analyzing the full-length sequences of 28 genotype- 4 and 11 genotype-3 HEV retrievable from DNA databases and 35 partial sequences recovered from patients with acute or fulminant hepatitis, we show that the presence of both U3148 and C5907 is associated with fulminant hepatitis in patients with HEV of genotype $4(p=0.0042)$ and genotype 3 or $4(p=0.0009)$, and that the prothrombin activity is significantly lower in patients infected with HEV carrying U3148 and C5907 than in those without the substitutions $(p=0.0069)$. U3148 and C5907 are silent substitutions that do not change amino acid. However, since U3148 is located at the RNA helicase domain and C5907 is located within the capsid gene, the secondary structure of the HEV RNA genome carrying U3148 and C5907 may be favorable for translation of the viral proteins. C5907 was associated with high HEV load $\left(\geq 10^{5} \mathrm{copies} / \mathrm{ml}\right)$ at initial examination $(p=0.0427)$. We propose that U3148 and C5907 are associated with the severity of hepatitis $\mathrm{E}$. hepatitis E virus; genotype; full-length sequence; U3148; C5907.

Tohoku J. Exp. Med., 2009, 218 (4), 279-284. (C) 2009 Tohoku University Medical Press

Hepatitis E, which is caused by hepatitis E virus (HEV), occurs in many parts of the world. HEV is transmitted via both the fecal-oral route and the zoonotic food-borne route (Okamoto et al. 2003). Several species of animals such as swine are considered to serve as reservoirs of HEV (Meng et al. 1998; Takahashi et al. 2003), and ingestion of uncooked or undercooked viscera of these animals may be the major route of HEV infection in industrialized countries including Japan (Yazaki et al. 2003).

HEV is a single-stranded, positive-sense RNA virus without an envelope and is currently classified as the sole member of the genus Hepevirus in the family Hepeviridae. The genome of HEV is approximately 7.2 kilobases in length and contains three open reading frames (ORF1, ORF2, and ORF3) flanked by short untranslated regions. HEV sequences have been classified into four genotypes (genotypes 1-4) based on the nucleotide sequence heterogeneity of HEV (Schlauder and Mushahwar 2001). HEV genotypes 1 and 2 were responsible for waterborne epidemics of HEV infection in Asia, Africa, and Mexico. Genotype 3 has been found worldwide, and genotype 4 was isolated in Asia (Okamoto 2007); HEV genotypes 3 and 4 are considered to undergo zoonotic transmission based on the close relationship between HEV isolates from humans and those from animals (Meng et al. 1997; Nishizawa et al. 2003; Tei et al. 2003; Yazaki et al. 2003).

Although hepatitis E usually resolves without specific therapy in most cases, it can progress to fulminant hepatitis. HEV is a major cause of fulminant hepatitis in endemic areas of Asia such as India and Bangladesh (Sheikh et al. 2002). To date, 7 of 9 HEV isolates obtained from fulminant hepatitis patients in Japan belonged to genotype 4,

Received May 12, 2009; revision accepted for publication June 9, 2009. doi: 10.1620/tjem.218.279

The nucleotide sequences data reported in this study have been assigned GenBank/EMBL/DDBJ accession numbers AB476428AB476462.

Correspondence: Hiroaki Okamoto, M.D., Ph.D., Division of Virology, Department of Infection and Immunity, Jichi Medical University School of Medicine, 3311-1 Yakushiji, Shimotsuke-Shi, Tochigi-Ken 329-0498, Japan.

e-mail: hokamoto@jichi.ac.jp 
although a larger number of hepatitis E patients in Japan are infected with genotype $3 \mathrm{HEV}$ in Japan (Okamoto et al. 2003), and the association of genotype $4 \mathrm{HEV}$ with fulminant hepatitis was suggested. The association between the nucleotide substitution of $U$ at nucleotide (nt) 3148 (U3148) in HEV genotype 4 and fulminant hepatitis was also suggested (Inoue et al. 2006). However, mutations in the viral genome associated with the development of fulminant hepatitis E are not fully understood, and extensive studies with a large number of samples are needed. Therefore, in the present study, we studied additional nucleotide substitutions that may be associated with the occurrence of fulminant hepatitis and disease severity.

\section{Materials and Methods}

\section{Full-length sequences of HEV genomes}

Full-length nucleotide sequences of 28 genotype $4 \mathrm{HEV}$ isolates recovered from patients with acute hepatitis $(n=23)$ or fulminant hepatitis $(n=5)$ and 11 genotype $3 \mathrm{HEV}$ isolates recovered from patients with acute hepatitis $(n=10)$ or fulminant hepatitis $(n=1)$, were retrieved from the GenBank/EMBL/DDBJ databases.

\section{Serum samples}

Serum samples that had been collected at the initial examinations [0-19 days (mean, 7.2 days) after disease onset] between 1993 and 2004 from 36 hepatitis E patients in Japan were subjected to determination of the partial sequence of ORF2 in the HEV genome containing nt 5907. Twenty of the patients [including one patient with fulminant hepatitis: HE-JF2 (Suzuki et al. 2002)] were infected with genotype $3 \mathrm{HEV}$, and 16 patients [including one patient with fulminant hepatitis: HE-JF1 (Suzuki et al. 2002)] were infected with genotype $4 \mathrm{HEV}$. All of these samples were used in a previous study to determine the partial sequence containing nt 3148 (Inoue et al. 2006). The study protocol was approved by the Ethics Committees in the institutions.

Amplification of partial nucleotide sequences of ORF2

Total RNA was extracted from $50 \mu 1$ of serum and subjected to reverse transcription (RT) with antisense primer HE344 [5' $-\mathrm{TTN}$ ACN GTY GGC TCG CCA TTG GC-3' $(\mathrm{N}=\mathrm{A}, \mathrm{C}, \mathrm{G}$, or T; $\mathrm{Y}=\mathrm{C}$ or $\mathrm{T})$ ]. The resulting CDNA was subjected to nested polymerase chain reaction (PCR) with genotype-specific primers to amplify the 514-nt sequence within ORF2 (nt 5825-6338; primer sequences at both ends excluded) [nucleotide numbers are in accordance with HE-JA1 (accession no. AB097812)]. For genotype $3 \mathrm{HEV}$, the first-round PCR was carried out with primers HE461 (5'-ACR ATC CGT TAY CGC CCG YTG GT-3') and HE344, and the second round was carried out with primers HE462 (5'-TAT GCT ATY TCY ATY TCY TTY TGG C-3') and HE343 (5'-CCR GCC GAC GAA ATY AAT TCT GTC-3'). For genotype 4 HEV, HE464 (5'-GGT AYC GYC CRC TNG TGC CGA-3') and HE344 were used in the first round, and HE465 (5'-CGC YAT ATC TAT YTC TTT CTG GC-3') and HE343 in the second round.

To avoid contamination during PCR procedures, the guidelines of Kwok and Higuchi (1989) were strictly observed. Two negative controls and one positive control were included for every 17 test samples. Results were recorded only when false-positive results were not obtained for the negative controls and HEV RNA was detected in the positive control. The negative control was water treated in the same way as the serum samples. The positive control was serum from a Nepali patient with a sporadic case of acute hepatitis E caused by HEV of genotype 1 (Shrestha et al. 2003), used at a dilution of $1: 1,000$ in anti-HEV-negative human sera obtained from healthy individuals.

\section{Sequence analysis}

The amplification products were sequenced on both strands directly using the BigDye Terminator v3.1 Cycle Sequencing kit on an ABI PRISM 3100 Genetic Analyzer (Applied Biosystems, Foster City, CA). Sequence analysis was performed using Genetyx-Mac (Version 12.2.7; Genetyx Corp., Tokyo, Japan).

\section{Quantification of HEV RNA}

Quantification of HEV RNA in serum samples was performed by real-time detection RT-PCR as described previously (Takahashi et al. 2008), using a QuantiTect Probe RT-PCR Kit (QIAGEN K.K., Tokyo, Japan) in a LightCycler apparatus (Roche Diagnostics K.K., Tokyo, Japan).

\section{Statistical analysis}

Statistical analyses were performed using Fisher's exact probability test for comparison of proportions between two groups and the Mann-Whitney U test for comparison of continuous variables between two groups. Differences were considered to be statistically significant when $p<0.05$.

\section{Results \\ Analysis of full-length genome of genotype $4 \mathrm{HEV}$ isolates}

First, 28 full-length nucleotide sequences of genotype $4 \mathrm{HEV}$ that were retrievable from the GenBank/EMBL/ DDBJ databases, were compared to narrow the targets of nucleotides that might be associated with the occurrence of fulminant hepatitis. When 5 isolates obtained from fulminant hepatitis patients and 23 isolates obtained from acute hepatitis patients were compared throughout the genome, substitutions at 7 nucleotides of nt $370,1816,3185,3856$, 4579,5907 , and 6126 were found to be significantly related to fulminant hepatitis. Among them, the substitution of $\mathrm{C}$ at nt 5907 (C5907) was most closely associated with fulminant hepatitis (100\% of fulminant hepatitis, $39.1 \%$ of acute hepatitis, $p=0.0204)$. Therefore, we determined partial sequences containing nt 5907 in 35 additional isolates, and analyzed the relationship between C5907 and fulminant hepatitis or disease severity. The substitution, U3148, which was found to be associated with the development of fulminant hepatitis in our previous study (Inoue et al. 2006), was also analyzed. Upon nested RT-PCR of 36 samples to amplify nt 5825-6338, all but one were successfully amplified and the sequences of the amplicons were analyzed: one genotype 4 sample (HE-JA31) could not be amplified due to low HEV load.

Comparison of frequency of C5907 and U3148 among genotype 3 and $4 \mathrm{HEV}$ isolates

The analysis of a total of $74 \mathrm{HEV}$ isolates [39 full- 
Table 1. Nucleotide substitutions of hepatitis E virus genomes associated with fulminant hepatitis.

\begin{tabular}{|c|c|c|c|c|c|c|}
\hline & \multicolumn{3}{|c|}{ Genotype $4 \mathrm{HEV}$ isolates with: } & \multicolumn{3}{|c|}{ Genotype 3 and $4 \mathrm{HEV}$ isolates with: } \\
\hline & U3148 & C5907 & U3148 and C5907 & $\mathrm{U} 3148$ & C5907 & U3148 and C5907 \\
\hline Fulminant hepatitis & $\begin{array}{c}6 / 6 \\
(100 \%)\end{array}$ & $\begin{array}{c}5 / 6 \\
(83.3 \%)\end{array}$ & $\begin{array}{c}5 / 6 \\
(83.3 \%)\end{array}$ & $\begin{array}{c}6 / 7 \\
(85.7 \%)\end{array}$ & $\begin{array}{c}5 / 7 \\
(71.4 \%)\end{array}$ & $\begin{array}{c}5 / 7 \\
(71.4 \%)\end{array}$ \\
\hline Acute hepatitis & $\begin{array}{c}15 / 37 \\
(40.5 \%)\end{array}$ & $\begin{array}{c}17 / 37 \\
(45.9 \%)\end{array}$ & $\begin{array}{c}7 / 37 \\
(18.9 \%)\end{array}$ & $\begin{array}{c}17 / 67 \\
(25.4 \%)\end{array}$ & $\begin{array}{c}23 / 67 \\
(34.3 \%)\end{array}$ & $\begin{array}{c}7 / 67 \\
(10.4 \%)\end{array}$ \\
\hline$p$ & 0.0089 & 0.1029 & 0.0042 & 0.0030 & 0.0668 & 0.0009 \\
\hline
\end{tabular}

U3148, $\mathrm{U}$ at nucleotide position of 3148; C5907, C at nucleotide position of 5907.

The frequency of U3148, C5907, and the combination of U3148 and C5907 was compared among 43 HEV isolates of genotype 4 recovered from 6 patients with fulminant hepatitis E and 37 patients with acute hepatitis E as well as among 74 HEV isolates of genotypes 3 and 4 recovered from 7 patients with fulminant hepatitis $E$ and 67 patients with acute hepatitis E.
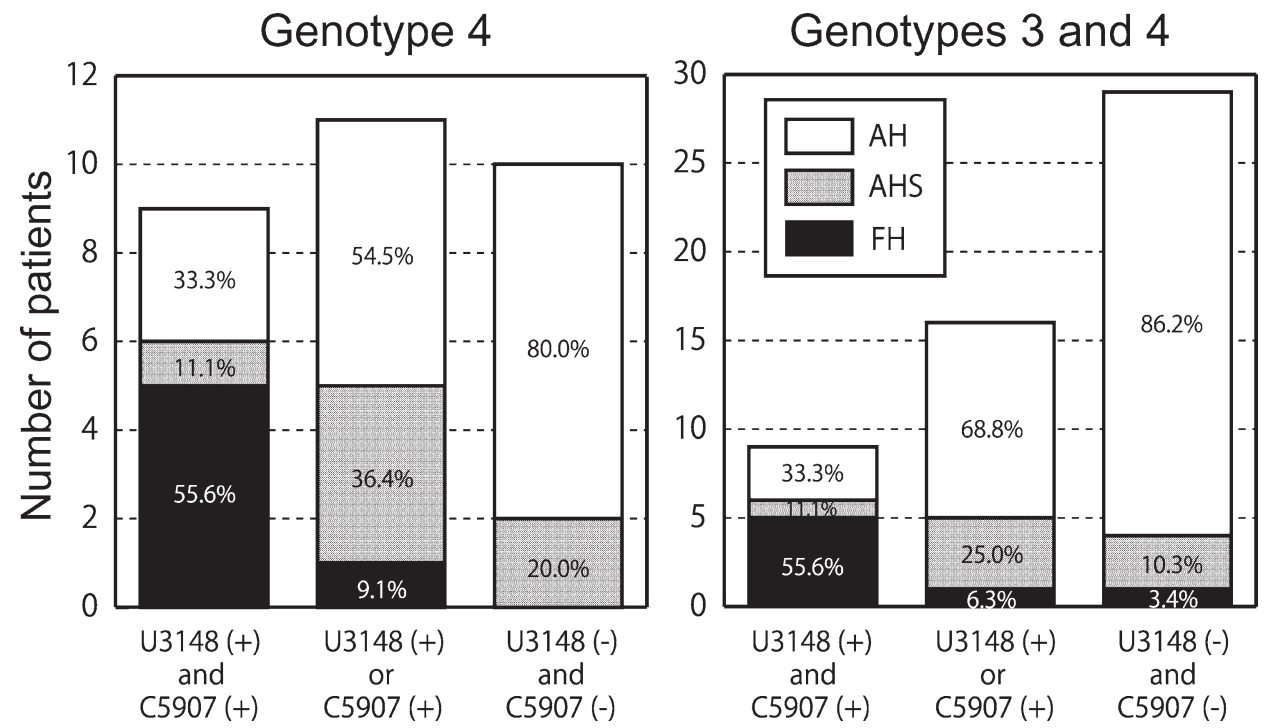

Fig. 1. The association of the nucleotide substitutions of the HEV genome and disease severity.

The frequencies of fulminant hepatitis ( $\mathrm{FH})$, the severe form of acute hepatitis (AHS), and acute hepatitis (AH) were compared in 3 groups with or without U3148 and C5907 among patients with genotype 4 HEV (Genotype 4) and among those with genotype 3 or 4 (Genotypes 3 and 4).

length sequences (28 genotype 4 and 11 genotype 3 ) retrievable from Genbank/EMBL/DDBJ and 35 partial sequences (15 genotype 4 and 20 genotype 3 ) determined in the present study] revealed that the frequency of C5907 was not significantly different between fulminant hepatitis and acute hepatitis in HEV of genotype $4(n=43)$ or genotype 3 or 4 (overall, $n=74$ ) (Table 1), although U3148 was significantly more frequent in fulminant hepatitis than in acute hepatitis $[p=0.0089$ (genotype 4) and $p=0.0030$ (overall)]. When the frequency of the co-occurrence of U3148 and C5907 was compared, these substitutions in genotype 4 HEV were found significantly more frequently in fulminant hepatitis than in acute hepatitis $(p=0.0042)$, with a similar situation in genotype 3 and $4 \mathrm{HEV}(p=0.0009)$.

\section{Comparison of disease severity according to nucleotide substitutions of HEV}

To evaluate the association of the nucleotide substitutions and disease severity, we analyzed clinical features and viral markers of 30 patients with genotype $4 \mathrm{HEV}$ and 24 patients with genotype $3 \mathrm{HEV}$, in whom such data were available. The patterns of the nucleotide substitutions were separated into 3 groups: U3148 (+) and C5907 (+) group, U3148 (+) or C5907 (+) group, and U3148 (-) and C5907 (-) group: age (mean, 60.3, 53.6, and 58.9 years, respectively) and sex (male, $100 \%, 87.5 \%$, and $82.8 \%$, respectively) were not significantly different among the three groups. Upon comparison of the numbers of patients with fulminant hepatitis, the severe form of acute hepatitis [lowest prothrombin time (PT) $\leq 40 \%$ without encephalopathy), and acute hepatitis (Fig. 1), fulminant hepatitis was significantly more frequent in the U3148 (+) and C5907 (+) group than in the other two groups when both genotype $4[55.6 \%$ vs. $9.1 \%(p=0.0379) ; 55.6 \%$ vs. $0 \%(p=0.0108)]$ and genotypes 3 and $4[55.6 \%$ vs. $6.3 \%$ ( $p=0.0119) ; 55.6 \%$ vs. $3.4 \%$ $(p=0.0014)]$ were analyzed. The severe form of acute hepatitis was seen frequently in the U3148 (+) or C5907 (+) group, and the acute hepatitis patients tended to be more 


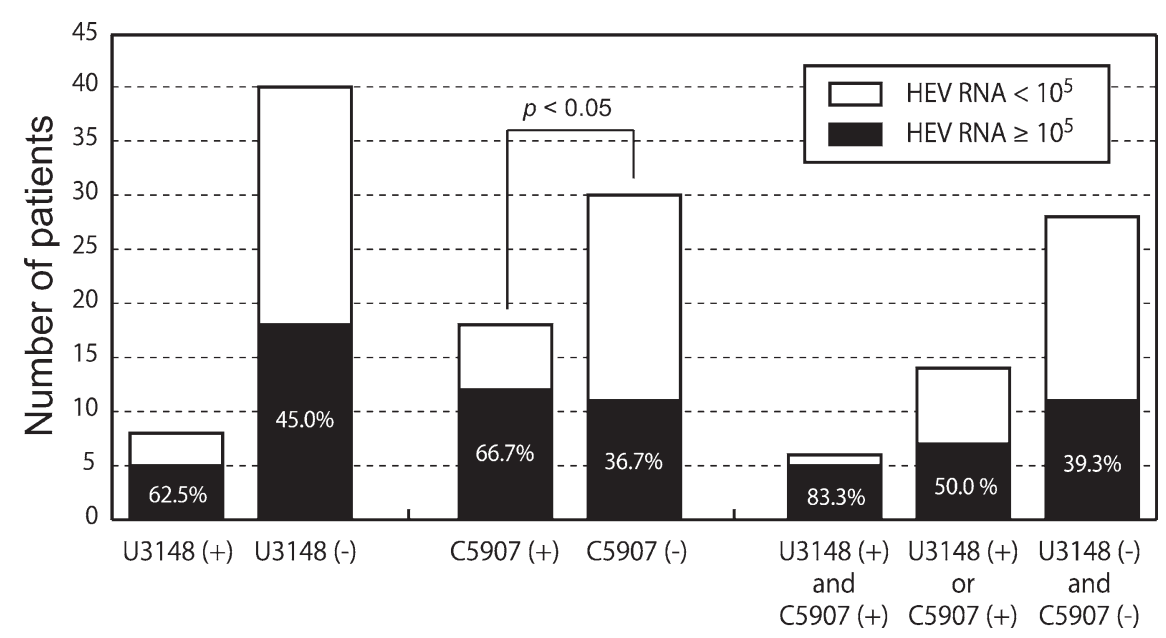

Fig. 2. The relationship between the HEV load at initial examination and the nucleotide substitutions.

The frequency of hepatitis E patients with high HEV load $\left(\geq 10^{5}\right.$ copies/ml) at initial examination, was compared between two groups with or without U3148, between two groups with or without C5907, or among 3 groups [U3148 (+) and C5907 (+) group ( $n=6)$, U3148 (+) or C5907 (+) group $(n=14)$, and U3148 (-) and C5907 (-) group $(n=28)]$.

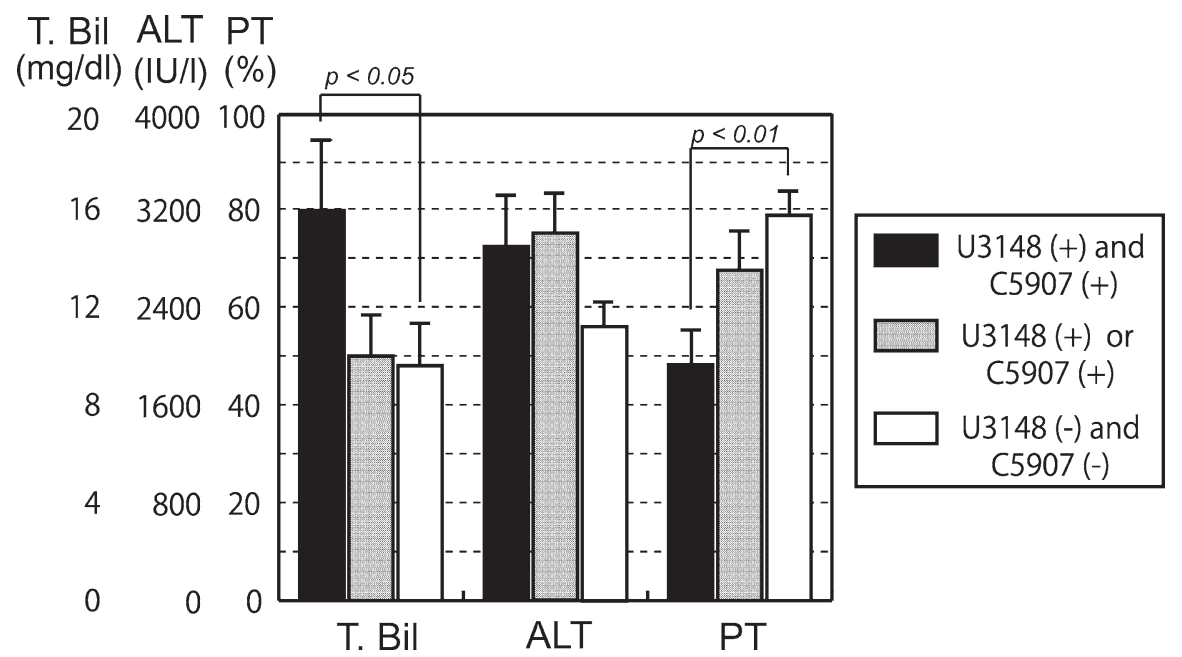

Fig. 3. Comparison of laboratory data among 3 groups with or without U3148 and C5907.

Peak values of total bilirubin (T. Bil, mg/dl) and alanine aminotransferase (ALT, IU/l) and the lowest prothrombin activity (PT, \%) were compared among 3 groups [U3148 (+) and C5907 (+) group $(n=9)$, U3148 (+) or C5907 (+) group $(n=$ $16)$, and U3148 (-) and C5907 (-) group $(n=29)]$. The error bar indicates the standard error of the mean.

frequent in the U3148 (-) and C5907 (-) group, although the differences were not statistically significant.

\section{Association between HEV nucleotide substitutions and viral load or laboratory data}

The relationship between the HEV load at the initial examination and the nucleotide substitutions was assessed. Patients with high HEV load $\left(\geq 10^{5}\right.$ copies $\left./ \mathrm{ml}\right)$ were significantly more frequent in the C5907 (+) group than in the C5907 (-) group $(66.7 \%$ vs. $36.7 \%, p=0.0427$ ) (Fig. 2), although the number of days after disease onset when the HEV load was quantified was nearly equal between the two groups (median, 7.5 vs. 6.5 days; $p=0.4939$ ). The viral load did not significantly differ between any of the substitution combinations as indicated in Fig. 2.

Laboratory data of the hepatitis $\mathrm{E}$ patients were ana- lyzed in relation to the nucleotide substitution patterns (Fig. 3). When the peak value of total bilirubin (T. Bil), the peak value of alanine aminotransferase (ALT), and the lowest PT activity were analyzed, T. Bil was significantly higher in the U3148 (+) and C5907 (+) group than in the U3148 (-) and C5908 (-) group ( $p=0.0352)$, and, conversely, PT was significantly lower in the former group than in the latter group $(p=0.0069)$. Although there was no appreciable difference, the ALT tended to be lower in the U3148 (-) and C5907 (-) group than in the other two groups.

\section{Discussion}

It was believed that the severity of hepatitis $\mathrm{E}$ was associated with host factors such as pregnancy and aging (Harrison 1999; Purcell et al. 2001). A recent report suggested that the mortality of pregnant patients with HEV- 
related acute liver failure was not increased compared with the mortality of pregnant patients with non-HEV-related acute liver failure, although HEV-related acute liver failure was more common than acute liver failure with other etiologies during pregnancy in India (Bhatia et al. 2008). As for viral factors, previous studies revealed that genotype $4 \mathrm{HEV}$ is more strongly associated with the severe form of hepatitis E than genotype $3 \mathrm{HEV}$ (Mizuo et al. 2005; Ohnishi et al. 2006) and that the nucleotide substitution of U3148 was associated with fulminant hepatitis based on comparison of full-length sequences of genotype $4 \mathrm{HEV}$ (Inoue et al. 2006). Because U3148 is a silent substitution that does not change the amino acid, the underlying mechanism for the association of U3148 with the progression to fulminant hepatitis remains unknown. The conservation of $22 \mathrm{nt}$ around nt 3148 in all four HEV genotypes led us to speculate that the nucleotide sequence containing nt 3148 might have functions at the nucleotide level.

The present study revealed that another nucleotide substitution at nt 5907 (C5907) was more prevalent in fulminant hepatitis patients than in acute hepatitis patients who were infected with HEV strains of genotype 3 or 4 , and that the presence of both U3148 and C5907 in HEV strains of genotype 3 or 4 was significantly associated with the occurrence of fulminant hepatitis and disease severity. Furthermore, C5907 had a significant association with high viral load ( $\geq 10^{5}$ copies $/ \mathrm{ml}$ ) of HEV of genotype 3 or 4 at the initial examination, although the viral load did not statistically differ between patients with or without C5907.

Kar et al. (2008) reported that the viral load of genotype $1 \mathrm{HEV}$ was significantly higher in pregnant patients than in nonpregnant patients, and the high viral load might lead to severe liver disease. Genotype 3 and 4 HEV strains with C5907 and/or U3148 might replicate efficiently and likely enhances the inflammation in the liver. The underlying mechanism by which C5907 heightens the replicative capability of HEV is unknown, because it is a silent substitution as is U3148. Nt 5907 locates within ORF2, and there is no subgenomic mRNA that initiates downstream of nt 5907. However, there is a possibility that the secondary structure of the RNA genome with C5907 may be favorable for translation of the capsid protein encoded by ORF2, as discussed previously (Hirata et al. 2003). Since nt 5907 is distant from nt 3148 and these nucleotides locate within different ORFs, C5907 and U3148 may act independently to enhance viral replication. An infectious cDNA clone of HEV established recently (Yamada et al. 2009) would be useful for investigating whether U3148 and C5907 actually affect the replication capacity of HEV in a genotype-dependent manner or not.

Since the number of samples from fulminant hepatitis patients was small (6 patients with genotype $4 \mathrm{HEV}, 1$ patient with genotype $3 \mathrm{HEV}$ ) in the current study, further accumulation of cases with hepatitis $\mathrm{E}$, especially fulminant hepatitis E, is needed. Although it was reported that HEV infection is the cause of $10-20 \%$ of acute or fulminant hepa- titis cases with non-A, non-B, non-C etiology in Japan (Suzuki et al. 2002; Okamoto et al. 2003), measurement of HEV markers is not approved by public medical insurance in Japan to date. We believe that testing for hepatitis E should be routine in this country, in cases of suspected viral hepatitis.

In conclusion, this study suggested that the silent substitutions of U3148 and C5907 in the genotype 3 and 4 HEV strains are closely associated with the occurrence of fulminant hepatitis and severity of hepatitis, and that C5907 is associated with high HEV load. The underlying mechanism has to be clarified in future studies in vitro, with the advent of a reverse genetics system for HEV (Yamada et al. 2009).

\section{Acknowledgments}

This study was supported in part by grants from Ministry of Education, Culture, Sports, Science, and Technology of Japan; Ministry of Health, Labor, and Welfare of Japan.

\section{References}

Bhatia, V., Singhal, A., Panda, S.K. \& Acharya, S.K. (2008) A 20-year single-center experience with acute liver failure during pregnancy: is the prognosis really worse? Hepatology, 48, $1577-1585$.

Harrison, T.J. (1999) Hepatitis E virus - an update. Liver, 19, 171-176.

Hirata, H., Lu, X., Yamaji, Y., Kagiwada, S., Ugaki, M. \& Namba, S. (2003) A single silent substitution in the genome of Apple stem grooving virus causes symptom attenuation. J. Gen. Virol., 84, 2579-2583.

Inoue, J., Nishizawa, T., Takahashi, M., Aikawa, T., Mizuo, H., Suzuki, K., Shimosegawa, T. \& Okamoto, H. (2006) Analysis of the full-length genome of genotype 4 hepatitis $\mathrm{E}$ virus isolates from patients with fulminant or acute self-limited hepatitis E. J. Med. Virol., 78, 476-484.

Kar, P., Jilani, N., Husain, S.A., Pasha, S.T., Anand, R., Rai, A. \& Das, B.C. (2008) Does hepatitis E viral load and genotypes influence the final outcome of acute liver failure during pregnancy? Am. J. Gastroenterol., 103, 2495-2501.

Kwok, S. \& Higuchi, R. (1989) Avoiding false positives with PCR. Nature, 339, 237-238.

Meng, X.J., Halbur, P.G., Shapiro, M.S., Govindarajan, S., Bruna, J.D., Mushahwar, I.K., Purcell, R.H. \& Emerson, S.U. (1998) Genetic and experimental evidence for cross-species infection by swine hepatitis E virus. J. Virol., 72, 9714-9721.

Meng, X.J., Purcell, R.H., Halbur, P.G., Lehman, J.R., Webb, D.M., Tsareva, T.S., Haynes, J.S., Thacker, B.J. \& Emerson, S.U. (1997) A novel virus in swine is closely related to the human hepatitis E virus. Proc. Natl. Acad. Sci. U.S.A., 94, 9860-9865.

Mizuo, H., Yazaki, Y., Sugawara, K., Tsuda, F., Takahashi, M., Nishizawa, T. \& Okamoto, H. (2005) Possible risk factors for the transmission of hepatitis $\mathrm{E}$ virus and for the severe form of hepatitis E acquired locally in Hokkaido, Japan. J. Med. Virol., 76, 341-349.

Nishizawa, T., Takahashi, M., Mizuo, H., Miyajima, H., Gotanda, Y. \& Okamoto, H. (2003) Characterization of Japanese swine and human hepatitis E virus isolates of genotype IV with $99 \%$ identity over the entire genome. J. Gen. Virol., 84, 1245-1251.

Ohnishi, S., Kang, J.H., Maekubo, H., Arakawa, T., Karino, Y., Toyota, J., Takahashi, K. \& Mishiro, S. (2006) Comparison of clinical features of acute hepatitis caused by hepatitis E virus (HEV) genotypes 3 and 4 in Sapporo, Japan. Hepatol. Res., 36, 301-307. 
Okamoto, H. (2007) Genetic variability and evolution of hepatitis E virus. Virus Res., 127, 216-228.

Okamoto, H., Takahashi, M. \& Nishizawa, T. (2003) Features of hepatitis $\mathrm{E}$ virus infection in Japan. Intern. Med., 42, 1065-1071.

Purcell, R.H. \& Emerson, S.U. (2001). Hepatitis E virus. In Fields Virology, 4th ed., edited by D.M. Knipe, P.M. Howley, D.E. Griffin, R.A. Lamb, M.A. Martin, B. Roizman \& S.E. Straus. Lippincott Williams \& Wilkins, Philadelphia, PA, pp. 30513061 .

Schlauder, G.G. \& Mushahwar, I.K. (2001) Genetic heterogeneity of hepatitis E virus. J. Med. Virol., 65, 282-292.

Sheikh, A., Sugitani, M., Kinukawa, N., Moriyama, M., Arakawa, Y., Komiyama, K., Li, T.C., Takeda, N., Ishaque, S.M., Hasan, M. \& Suzuki, K. (2002) Hepatitis e virus infection in fulminant hepatitis patients and an apparently healthy population in Bangladesh. Am. J. Trop. Med. Hyg., 66, 721-724.

Shrestha, S.M., Shrestha, S., Tsuda, F., Nishizawa, T., Gotanda, Y., Takeda, N. \& Okamoto H. (2003) Molecular investigation of hepatitis $\mathrm{E}$ virus infection in patients with acute hepatitis in Kathmandu, Nepal. J. Med. Virol., 69, 207-214.

Suzuki, K., Aikawa, T. \& Okamoto, H. (2002) Fulminant hepatitis E in Japan. N. Engl. J. Med., 347, 1456.
Takahashi, M., Nishizawa, T., Miyajima, H., Gotanda, Y., Iita, T., Tsuda, F. \& Okamoto, H. (2003) Swine hepatitis E virus strains in Japan form four phylogenetic clusters comparable with those of Japanese isolates of human hepatitis $\mathrm{E}$ virus. $J$. Gen. Virol., 84, 851-862.

Takahashi, M., Yamada, K., Hoshino, Y., Takahashi, H., Ichiyama, K., Tanaka, T. \& Okamoto, H. (2008) Monoclonal antibodies raised against the ORF3 protein of hepatitis E virus (HEV) can capture HEV particles in culture supernatant and serum but not those in feces. Arch. Virol., 153, 1703-1713.

Tei, S., Kitajima, N., Takahashi, K. \& Mishiro, S. (2003) Zoonotic transmission of hepatitis $\mathrm{E}$ virus from deer to human beings. Lancet, 362, 371-373.

Yamada, K., Takahashi, M., Hoshino, Y., Takahashi, H., Ichiyama, K., Tanaka, T. \& Okamoto, H. (2009) Construction of an infectious cDNA clone of hepatitis E virus strain JE03-1760F that can propagate efficiently in cultured cells. J. Gen. Virol., 90, 457-462.

Yazaki, Y., Mizuo, H., Takahashi, M., Nishizawa, T., Sasaki, N., Gotanda, Y. \& Okamoto, H. (2003) Sporadic acute or fulminant hepatitis E in Hokkaido, Japan, may be food-borne, as suggested by the presence of hepatitis $\mathrm{E}$ virus in pig liver as food. J. Gen. Virol., 84, 2351-2357. 